\title{
One Snapshot DOA Estimation Method using Two Elements Antenna Array
}

\author{
Han Trong Thanh, An Trung Hung, Nguyen Thi Le Quyen, Pham Van Hieu, Do Trinh Sa \\ School of Electronics and Telecommunications, Hanoi University of Science and Technology, \\ No.1, Dai Co Viet Road, Hai Ba Trung District, Hanoi, VIETNAM
}

\begin{abstract}
In this paper, a robust Radio Direction Finding (RDF) system which using 2 - elements antenna array and the Total Forward - Backward Matrix Pencil (TFBMP) is proposed. The simulation results for DOA estimation using proposed system will be assessed and analyzed to verify its performance.
\end{abstract}

\section{Keywords}

Radio Direction Finding - RDF, Direction Of Arrival - DOA, Total Forward - Backward Matrix Pencil - TFBMP, Software Defined Radio - SDR.

\section{INTRODUCTION}

Radio Direction Finding (RDF) system plays an important role in wireless communication in both civil and military area. In recent decades, Electronics devices are usually designed with smaller size with high processing speed. The dimension of products mainly depends on hardware part while the performance of devices is always depended on signal processing algorithm.

In practical, the size of antenna directly affects to the size of RDF system. There are many kinds of antenna array have been investigated and deployed in RDF system such as ULA, UCA, NLA, RLA due to their simplicity and convenient mathematical model for array processing... However, they are usually cumbersome. Therefore, it is very difficult to reduces the RDF system's size. In [1], a variant of an ULA model called Nested Antenna Array (NAA) was proposed. This model helps the RDF can estimate more number of DOA than number of antenna element. However, this is still not an optimal problem.

There are a lot of DOA estimation method were studied and developed. MUSIC [2] and ESPRIT [3] are the most famous super - resolution methods for DOA estimation which generally must calculate the signal covariance matrix. However, they cannot estimate the DOA with only one snapshot. In [4], a novel method that is faster and consumes less processing power than two above algorithms named Matrix Pencil (MP) was proposed. Especially, a remarkable advantage of this technique is that it can extract the DOA information with only one snapshot.

In [5-6], the Total Forward - Backward Matrix Pencil (TFBMP) is an extension of the Matrix Pencil Method was proposed. By using this method, the DOA information of the coherent incoming signals can be estimated accurately. The Total Forward - Backward is the pre - processing technique to break the correlative property of the received signals. Thus, this method need a larger database than the original method. However, this fact helps TFBMP method to estimate the DOA information of coherent incoming signals more efficient than other method. In [7], TFBMP was used for the high - resolution frequency estimator with the better estimation results than the other methods such as Fourier technique.

In this paper, a robust system using a 2 - element Antenna Array based on the TFBMP technique to estimate the DOA information is proposed. The performance of this method will be assessed in many cases that depend on the characteristics of incoming signals as well as antenna array properties.

The paper is organized as follows. Section II describes the structure of the 2 - element antenna array and its signal model. In section III, TFBMP technique for DOAs of those signals is presented in detail. The simulation results are shown in the section IV. The conclusion is given in the section V.

\section{ANTENNA STRUCTURE AND SIGNAL MODEL}

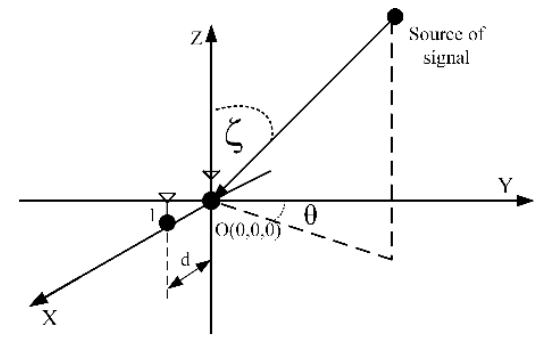

Fig. 1. Antenna Array in Cartesian coordinate system

In this paper, a 2 - element antenna array is used. This antenna is located in three dimensions Cartesian coordinate system as shown in Figure 1. Assume that the incoming signal at the far field of the array impinging on the ULA has DOA information in both elevation $(\xi)$ and azimuth $(\theta)$ as shown in Fig.1. However, in this work, only the signal in the same plane with antenna array is concerned. This means that the DOA of signal of interest is estimated in azimuth and $(\xi)=90^{\circ}$.

The phase at the $m^{\text {th }}$ antenna relative to the reference point is given as:

$\phi_{m}=\frac{2 \pi}{\lambda} m d \sin (\theta), m=(0,1)$

where $\lambda$ is the wavelength of signal of interested.

The phase at the $m^{\text {th }}$ antenna relative to the reference point is given as:

$a_{m}=g_{m} e^{j \phi_{m}}$

where $g_{m}$ is the gain of the $m^{\text {th }}$ antenna element.

The baseband output signal at the $m^{\text {th }}$ antenna is:

$$
x_{m}(\mathrm{t})=s(t) a_{m}=S(t) e^{j \frac{2 \pi}{\lambda} m d \sin (\theta)}
$$

where $s(t)$ is the incoming signal and $S(t)=s(t) \cdot g_{m}$. 
In practice, the antenna array can receive several radio signals simultaneously. The received signal at each antenna element will be the sum of all arriving radio signal. In case of $K$ signals from $K$ directions $\theta_{1}, \theta_{2}, \ldots, \theta_{K}$, respectively. The received signal in AWGN channel at the $m^{\text {th }}$ antenna element is:

$$
\begin{aligned}
x_{m}(t) & =\sum_{i=1}^{K} S_{i}(t) e^{j \beta m d \sin \left(\theta_{i}\right)}+\eta_{m} \\
& =\sum_{i=1}^{K} S_{i}(t) \cdot \alpha_{i}^{m}+\eta_{m}
\end{aligned}
$$

where $\beta=\frac{2 \pi}{\lambda}$ is the propagation factor, $\alpha_{i}=e^{j \beta m d \sin \left(\theta_{i}\right)}$ and $\eta_{m}$ is Gaussian noise at each antenna element.

According to Eq.2, the steering vector or manifold vector in each DOA $-\theta$ is defined as:

$\boldsymbol{a}(\theta)=\left[\begin{array}{ll}e^{j \phi_{0}} & e^{j \phi_{1}}\end{array}\right]^{T}=\left[\begin{array}{ll}1 & e^{j \frac{2 \pi}{\lambda} d \sin (\theta)}\end{array}\right]^{T}$

in which $T$ denotes transpose matrix. It is easy to see that the DOA information of many sources cannot be calculate when only using this manifold vector. Thanks to Kronecker and Khatri - Rao product, the manifold as in Eq.5 can be converted to the form of longer ULA manifold.

Firstly, the definitions of Matrices Product will be briefly discussed as follow

Definitions: Given two matrices $A_{m \times n}$ and $B_{p \times q}$

- $\quad$ The Kronecker product [7] of A and B is a $m p$ rows and $n q$ columns matrix.

$A \otimes B=\left[\begin{array}{cccc}a_{11} B & a_{12} B & \ldots & a_{1 n} B \\ a_{21} B & a_{22} B & \ldots & a_{2 n} B \\ \vdots & \vdots & \vdots & \vdots \\ a_{m 1} B & a_{m 2} B & \ldots & a_{m n} B\end{array}\right]$

- Khatri - Rao Product of A and B is a mn rows and p columns matrix which is rewritten by the Kronecker product as the following

$$
A \odot B=\left[a_{1} \otimes b_{1} \mid a_{2} \otimes b_{2} \ldots a_{p} \otimes b_{p}\right]_{m n \times p}
$$

where " $\otimes$ " and “ $\odot$ ” denote Kronecker and Khatri - Rao product and $a_{1}, a_{2} \ldots a_{p}$ and $b_{1}, b_{2} \ldots b_{p}$ are the columns of matrixes $A$ and $B$, respectively.

Let us consider an array of $\mathrm{M}$ sensors, with $\vec{d}_{i}$ denoting the position vector of the $i^{\text {th }}$ sensor. Define the set

$$
D=\left\{\vec{d}_{i}-\vec{d}_{j}\right\} \text {, with } i, j=1 \div M
$$

In our definition of the set $D$, we allow repetition of its elements. We also define the set $D_{u}$ which consists of the distinct elements of the set $D$. Then, the difference co-array of the given array is defined as the array which has sensors located at positions given by the set $D_{u}$.

The number of elements in the difference array (given by the set $D_{u}$ ) directly decides the distinct values of the crosscorrelation terms in the covariance matrix of the signal received by an antenna array.

The difference array of an M - element ULA array is a filled ULA array with $2 M-1$ elements whose positions are given by the set $P_{c a}$ defined as

$$
D=\left\{\vec{d}_{i}-\vec{d}_{j}\right\}, \text { with } i, j=1 \div M
$$

In case of 2 element antenna array, the dimension of vitrual array manifold $A^{*} \odot A$ is $(M)^{2} \times K$. Where (*) denotes the complex conjugate matrix and $K$ is the number of incoming signals. A new matrix $\tilde{A}$ with size $2 M-1$ is constructed by removing the repeat rows from $A^{*} \odot A$ (after their first occurrence) and also sorting them so that the $i^{\text {th }}$ row corresponds to the element location $\left\{-M_{c a}+i\right\}$.

It can be seen that $\tilde{A}$ behaves like the manifold of a virtual ULA array (longer than original array) with $2 M-1$ elements. The position given by the distinct values of set $P_{c a}$. This array is precisely the difference array of the original array.

As above analysis, the new virtual array is the ULA array. Therefore, by using Khatri - Rao product continuously until the number of virtual antenna elements large enough, the RDF system can determine the direction of the $K$ sources.

Instead of working with the original antenna array, the DOA information can be calculated by using the new virtual ULA array with $M_{k r}$ elements given by

$$
M_{k r}=2 M_{k r-1}-1
$$

where $k r$ is number of times used $\mathrm{Kr}$ - product, and $M_{0}=2$.

The manifold vector as Eq.5 can be rewritten as

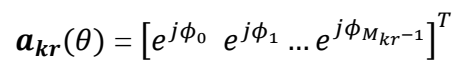

where $\phi_{m_{k r}}=\frac{2 \pi}{\lambda} m_{k r} d \sin (\theta)$ and $m_{k r}=-\frac{\left(M_{k r}-1\right)}{2} \div \frac{\left(M_{k r}-1\right)}{2}$

The discrete time output signal at $m^{\text {th }}$ element is

$$
x_{m}=\sum_{i=1}^{K} S_{i} \cdot e^{j \beta m_{k r_{m}} d \sin \left(\theta_{i}\right)}+\eta_{m}
$$

where $m=0,1,2 \ldots M_{k r}$.

\section{TOTAL FORWARD - BACKWARD MATRIX PENCIL METHOD FOR DOA ESTIMATION}

Base on TFBMPM, we have defined the matrices $Y_{0 f b}$ and $Y_{l f b}$ as:

$$
\begin{aligned}
Y_{0 f b_{2\left(M_{k r}-L\right) \times L}} & =\left[\begin{array}{ccccc}
z_{0} & z_{1} & \cdots & z_{L-2} & z_{L-1} \\
z_{L}^{*} & z_{L-1}^{*} & \cdots & z_{2}^{*} & z_{1}^{*}
\end{array}\right] \\
Y_{1 f b_{2\left(M_{k r}-L\right) \times L}} & =\left[\begin{array}{ccccc}
z_{1} & z_{2} & \cdots & z_{L-1} & z_{L} \\
z_{L-1}^{*} & z_{L-2}^{*} & \cdots & z_{1}^{*} & z_{0}^{*}
\end{array}\right]
\end{aligned}
$$

where ' $*$ ' denotes complex conjugate, $L$ is chosen as pencil parameter with the condition:

$$
K \leq L \leq M_{k r}-K+1
$$

and $z j(j=0, \ldots, L)$ is defined as

$$
\mathrm{z}_{j}^{T}=\left[\begin{array}{lllll}
x_{j} & x_{j+1} & \ldots & x_{M_{k r}-L+j-1}
\end{array}\right] ; j=0, \ldots, L
$$

Based on Eq.13 and Eq.14, all data matrix is constructed as:

$$
Y_{f b_{2\left(M_{k r}-L\right) \times(L+1)}}=\left[\begin{array}{ccccc}
z_{0} & z_{1} & \cdots & z_{L-1} & z_{L} \\
z_{L}^{*} & z_{L-1}^{*} & \cdots & z_{1}^{*} & z_{0}^{*}
\end{array}\right]
$$


In order to estimate the DOA information, the Singular Value Decomposition (SVD) of this matrix will be performed:

$$
\begin{aligned}
& Y_{f b_{2\left(M_{k r}-L\right) \times(L+1)}} \\
& U_{2\left(M_{k r}-L\right) \times 2\left(M_{k r}-L\right)} \Sigma_{2\left(M_{k r}-L\right) \times(L+1)} V_{(L+1) \times(L+1)}^{H}
\end{aligned}
$$

where $H$ denotes complex conjugate transpose of a matrix, $U$, $\Sigma$, and $V$ are given by

$$
\begin{gathered}
\Sigma=\operatorname{diag}\left\{\sigma_{1}, \sigma_{2}, \ldots, \sigma_{p}\right\} \\
p=\min \{2(\widetilde{M}-L), L+1\} \\
\sigma_{1} \geq \sigma_{2} \geq \ldots \geq \sigma_{p} \geq 0 \\
U=\left[u_{1}, u_{2}, \ldots, u_{2(\widetilde{M}-L)}\right] \\
Y_{f b}^{H} u_{i}=\sigma_{i} v_{i}, i=1, \ldots, p \\
V=\left[v_{1}, v_{2}, \ldots, v_{(L+1)}\right] \\
Y_{f b}^{H} v_{i}=\sigma_{i} u_{i}, i=1, \ldots, p \\
U^{H} U=I, V^{H} V=I
\end{gathered}
$$

$\sigma_{i}$ are the singular values of $Y_{f b}$ and the vector $u_{i}$ and $v_{i}$ are the $i^{\text {th }}$ left and right singular vector, respectively. In the next step, the $K$ largest singular values of $Y_{f b}$ can be achieved by using the singular value filtering.

$$
\bar{Y}_{f b_{2}(\widetilde{M}-L) \times(L+1)}=\bar{U}_{2(\widetilde{M}-L) \times K} \bar{\Sigma}_{K \times K} \bar{V}_{K \times(L+1)}^{H}
$$

where

$$
\bar{\Sigma}=\operatorname{diag}\left\{\sigma_{1}, \sigma_{2}, \ldots, \sigma_{K}\right\}
$$

has $K$ largest singular values of $\Sigma$, and the matrices $\bar{U}$ and $\bar{V}$ is $K$-truncation of $V$ :

$$
\bar{V}=\left[\bar{V}_{0}, v_{L+1}\right], \bar{V}=\left[v_{1}, \bar{V}_{1}\right]
$$

Similar to Eq.27, $\bar{Y}_{0 f b}$ and $\bar{Y}_{1 f b}$ are obtained as

$$
\bar{Y}_{0 f b}=\bar{U} \bar{\Sigma} \bar{V}_{0}^{H}, \bar{Y}_{1 f b}=\bar{U} \bar{\Sigma} \bar{V}_{1}^{H}
$$

Base on above equations, the matrix pencil can be established as

$$
M P=\bar{Y}_{1 f b}-z \bar{Y}_{0 f b}
$$

Left multiplying $M P$ by $\bar{Y}_{0 f b}^{+}$yields

$$
q^{H}\left(\bar{Y}_{1 f b} \bar{Y}_{0 f b}^{+}-z I\right)=0^{H}
$$

where $\bar{Y}_{0 f b}^{+}$is the Moore-Penrose pseudo inverse of $Y_{0 f b}$

$$
\bar{Y}_{0 f b}^{+}=\left(\bar{V}_{0}^{H}\right)^{+} \bar{\Sigma}^{-1} \bar{U}^{+}
$$

Substituting Eq.30 and Eq.33 into Eq.32 the equivalent generalized Eigen-problem becomes

$q^{H}\left(\bar{V}_{1}^{H}-z \bar{V}_{0}^{H}\right)=0^{H}$

By left multiplying by $\overline{V_{0}}$, Eq.34 becomes

$$
q^{H}\left(\bar{V}_{1}^{H} \bar{V}_{0}-z \bar{V}_{0}^{H} \bar{V}_{0}\right)=0^{H}
$$

Using the values of the generalized eigenvalues, $z$, of Eq.35, DOA information of incoming signal can be numerical calculated as

$$
\theta_{i}=\sin ^{-1}\left[\frac{\Im\left[\ln \left(z_{i}\right)\right]}{\beta d_{1}}\right]
$$

where $\mathfrak{I}\left[\ln \left(z_{i}\right)\right]$ is the imaginary part $\ln \left(z_{i}\right)$.

\section{SIMULATION RESULTS}

The simulation results of proposed system using Matlab will be analyzed to asses its performance. In this work, in order to evaluate the accuracy of the simulation, the Root Mean Square Error (RMSE) is used. This parameter is defined as

$$
R M S E=\sqrt{\frac{\sum_{i=1}^{K}\left(x_{i}-x_{i}^{\prime}\right)^{2}}{K}}
$$

where $x_{i}$ is the expected value and $x_{i}^{\prime}$ is the estimated value of measurement object $i^{\text {th }}$ and $K$ is the number of measurement objects.

In the first simulation, assuming that there are 8 signals imping on the proposed antenna array $(M=2)$ in AWGN channel with $S N R=3 d B$. The simulation result is shown in Fig.2.

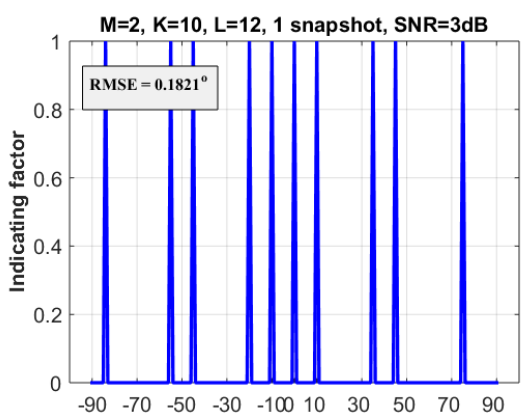

Angle in degrees(-85,-55,-45,-20,-10,0,10,35,45,75)

Fig. 2. Antenna Array in Cartesian coordinate system

It has to be noticed that the estimated DOAs in the simulation are the numerical values as in Eq.36. However, in order to demonstrate visually the result, it is illustrated in 2 dimension Cartesian coordinate system, in which the $\mathrm{X}$ - Axis is the DOA of incoming signals and the $\mathrm{Y}-$ Axis is indicating factor. This factor is set to 1 corresponding to the estimated DOA. Obviously, the proposed system has accurately estimated the DOA information of 10 incoming signals. Moreover, by using TFBMP, the DOA information can be calculated with only one snapshot. This is a significant advantage of TFPMP in comparison with other highresolution algorithms such as MUSIC. This issue helps to reduce considerably the sampling frequency as well as the amount of processing data. 


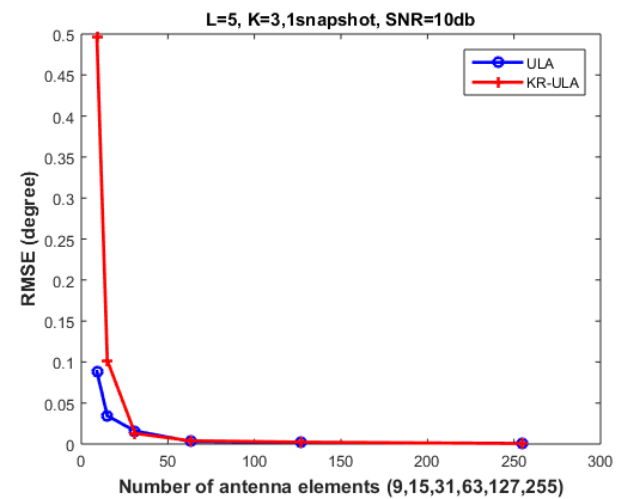

Fig. 3. DOA estimation accuracy of KR-ULA and ULA using TFBMP

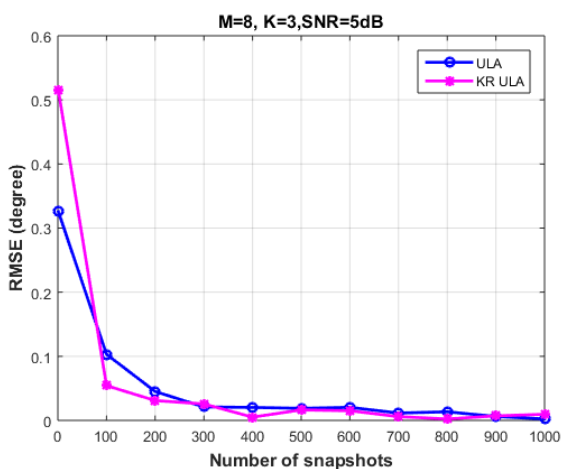

Fig. 4. DOA estimation accuracy and number of snapshot

In the second simulation, the performance of proposed system is compared to the performance of common RDF system which using ULA with varying number of elements. The simulation result plotted in the Fig.3 indicates that the accuracy of DOA estimation of proposed system is equivalent to common RDF system.

The relation between the accuracy of the algorithm and number of snapshots is shown in Fig.4. Based on the simulation result, it can be seen that when the number of snapshots is increased, the accuracy of this method will increase. And the accuracy of two systems are the same.

\section{CONCLUSIONS}

This research proposes a robust RDF system using 2 element antenna array and TFBMP algorithm. The proposed system can estimate more DOA information with only 2 - elements antenna array and one snapshot. Obviously, dimension of proposed system is smaller than other common systems. Moreover, with only one snapshot, the computation time as well as the sampling frequency and the size of buffer in real time receivers will be reduced significantly in comparison with other high-resolution DOA estimation algorithms.

\section{ACKNOWLEDGMENTS}

This research is carried out in the framework of the project funded by the Hanoi University of Science and Technology (HUST), Vietnam with the title "Research on Wideband DOA estimation algorithms for advance Radio Direction Finding System" under the grant number T2017-PC-113. The authors would like to thank the HUST for their financial support.

\section{REFERENCES}

[1] Pal, Piya, and P. P. Vaidyanathan. "Nested arrays: A novel approach to array processing with enhanced degrees of freedom." IEEE Transactions on Signal Processing 58.8 (2010), pp. 4167-4181.

[2] R. O. Schmidt, "Multiple emitter location and signal parameter estimation," IEEE Transactions on Antennas and Propagation, 34(3)(1986) 276-280.

[3] Ottersten B. and Kailath T. "Direction-of-arrival estimation for wide-band signals using the ESPRIT algorithm," IEEE Transactions on Acoustics, Speech and Signal Processing, vol. 38 (1990), pp. 317-327.

[4] Y. Hua and T. Sarkar, "Matrix pencil method for estimating parameters of exponentially damped/undamped sinusoids in noise," IEEE Transaction on Acoustics, Speech, and Signal Processing, 38(5)(1990) 814-824.

[5] J. E. F. del Rio and T. K. Sarkar, "Comparison between the matrix pencil method and the fourier transform technique for high-resolution spectral estimation," in Digital Signal Processing, 6(11)(1996) 108-125.

[6] Sales, Kirk L. Reducing the Number of Ultrasound Array Elements with the Matrix Pencil Method. Michigan State University. Electrical Engineering, 2012.

[7] Liu, Shuangzhe, and Gõtz Trenkler. "Hadamard, KhatriRao, Kronecker and other matrix products." Int. J. Inf. Syst. Sci 4.1 (2008), pp. 160-177. 